\title{
Isotopes in archaeology
}

\author{
Ricardo Fernandes $^{1,2} \cdot$ Klervia Jaouen $^{3}$
}

Published online: 16 May 2017

(C) Springer-Verlag Berlin Heidelberg 2017

Isotopic proxies have been employed within archaeological research since decades; however, their use has surged in recent years. Together with the increase in the number of published case studies, there have also been significant technical developments that improved greatly on available analytical techniques. Throughout the years, the introduction of novel isotopic proxies refined and expanded the existing knowledge on past environments and human activities. Such developments allowed for and were motivated by a growth of archaeological research topics. These have included, among others, climatic and environmental reconstruction, studies of past human diet, nutrition, and mobility, building accurate chronologies, past animal and crop management practices, pottery use, etc. Thus, an attempt at offering a complete overview of the applications and methodologies involved in isotopic analyses applied to archaeological research would represent an undertaking well beyond the limited scope of this special issue. Instead, this issue is aimed at highlighting a selection of special themes that represent a mix of well-established and recent topics within isotopic studies applied to archaeological research.

Ricardo Fernandes

rf385@cam.ac.uk; rfernandes@gshdl.uni-kiel.de

Klervia Jaouen

klervia_jaouen@eva.mpg.de

1 McDonald Institute for Archaeological Research, University of Cambridge, Downing St, Cambridge CB2 3ER, UK

2 Leibniz-Laboratory for Radiometric Dating and Isotope Research, University of Kiel, Max-Eyth-Str. 11-13, 24118 Kiel, Germany

3 Department of Human Evolution, Max Planck Institute for Evolutionary Anthropology, Deutscher Platz 6, 04103 Leipzig, Germany
There is an increasing recognition among the archaeoisotope community of the need to build isotopic baselines which establish the environmental isotopic signals for the past time periods and regions under study. An example of this is given by Knipper et al. that investigated the diets of humans from the late Iron Age burial site of Basel-Gasfabrik (Switzerland). Human diet reconstruction was done using a Bayesian mixing model and relied on stable isotope measurements on humans and a large amount of locally available archaeological faunal and botanical remains. The outcome of the study observed inter-individual differences in dietary intakes (e.g. millet consumption) although there were no significant gender differences. The emphasis on the need to employ archaeological baseline material contemporaneous with the period under study is suggested by the results from the study by Roffet-Salque et al. This study concerned pottery use which is often investigated through carbon stable isotope analysis of fatty acids recovered from the clay matrix of archaeological ceramic vessels. However, Roffet-Salque et al. showed that special care must be taken when employing modern fat references for archaeological studies, namely when modern animals are not fed only on purely natural pastures.

Essential to isotopic studies of diet and mobility is the understanding of how isotopic signals are transferred from the environment (e.g. water, foods) towards consumer tissues. This is often determined through animal and human feeding experiments. Also, studies that identify the importance of other effects (e.g. cooking) on isotopic signal transfer are relevant. Tuross et al. discuss the impact, for different parameters, that has to be considered when interpreting oxygen stable isotope ratios measured in human teeth and bones. Each human tissue has specific formation times and isotopic offsets towards oxygen pools. Furthermore, heating, through cooking, modifies the isotopic ratio of consumed water and foods. 
Three separate studies (Knipper et al., Pestle et al., Piličiauskas et al.) employed Bayesian mixing models to reconstruct past diets. These models include the multiple parameters involved in diet reconstruction and respective uncertainties (e.g. diet-to-tissue isotopic offsets, dietary routeing, etc) and offer a probabilistic interpretation of dietary intakes. Pestle et al. compared the dietary diversity of two populations, Solcor and Tchecar, in northern Chile dating to the Middle Period. Bayesian modelling of isotopic data showed that the Tchecar individuals had less varied diets than those from Solcor. The authors concluded that Middle Period individuals had a differentiated access to food resources challenging the hypothesis of uniform richness during the Middle Period. Another aspect of Bayesian modelling is the possibility of incorporating prior information from other sources of archaeological data. This was explored by Piličiauskas et al. that investigated the chronology of Subneolithic-Neolithic sites in Lithuania from human bone radiocarbon dates. Results from faunal studies were combined with carbon and nitrogen stable isotope measurements from the same bone material to quantify dietary intakes and correct for dietary radiocarbon reservoir effects. This allowed for a revised chronology of the region under study.

The study by Piličiauskas et al. also highlights the interconnection between diet and radiocarbon-based chronologies. In recent years, there have been several published case studies where significant human dietary radiocarbon reservoir effects were reported. This presents both a challenge and opportunity: a challenge since diet stable isotope studies become necessary for the establishment of accurate radiocarbon-based chronologies when relying on human remains and an opportunity since radiocarbon can increasingly be employed as a dietary proxy to detect the intake of aquatic foods. The contribution from Svyatko et al. showed that Eneolithic to Iron Age individuals from the Minusinsk Basin (Russia) have stable isotope values which could be consistent with freshwater fish intakes. However, radiocarbon dating of paired human and fauna overall showed an absence of significant dietary reservoir effects lending validity to previously proposed chronologies.

The interpretation of dietary or mobility reconstructions based on a limited number of isotopic proxies may remain ambiguous. This ambiguity may be reduced by the inclusion of additional isotopic proxies, that is, by including less commonly employed isotopic systems (e.g. sulphur isotopes) and/ or performing isotopic measurements on a larger variety of consumer tissues and/or molecular compounds (e.g. bone mineral, amino acids, fatty acids). In this respect, Jaouen and Pons discussed the potential of progressively employing nontraditional isotopic proxies (e.g. $\mathrm{Ca}, \mathrm{Cu}, \mathrm{Fe}, \mathrm{Mg}, \mathrm{Sr}, \mathrm{Zn}$ ), measured in mineral samples of bone and teeth, in diet reconstruction studies. Both the contributions from Pestle et al. and Díaz-del-Río et al. employed a multi-isotopic approach in the study of human diet or mobility. Díaz-del-Río et al. investigated the mobility of individuals from burial sites near Madrid (Spain) dating to the Late Neolithic, Chalcolithic, and Bronze Age using strontium and oxygen isotope ratios measured in teeth and bone. A minimum number of migrants could be identified. In addition, carbon isotope ratios suggest a dietary shift at c. $2500 \mathrm{cal} \mathrm{BC}$ towards the higher incorporation of $\mathrm{C} 4$ plant foods.

The study of the archaeology of the individual is linked to multi-proxy approaches. Previous case studies have reconstructed the isotopic life histories, often of high-status individuals, by taking isotopic measurements from multiple tissues corresponding to specific life periods of an individual. Jørkov and Gröcke performed a large diachronic study to investigate the diets of individuals buried at the Assistens cemetery in Copenhagen. Comparison of bone and hair stable isotope analysis allowed for interesting observations on nutritional status and dietary intakes across social groups and time. Kinaston and Buckley investigated intrapopulation variations in diet through carbon and nitrogen stable isotope analysis of bone and teeth of individuals from a $>300$-year-old burial ground at Taumako Island (Solomon Islands). The authors of the study found that dietary differences among adult males and females were not observed in children and that survival to adulthood was related to the consumption of higher trophic level foods.

The application of stable isotope research to archaeology is not limited to the study of human diet and mobility, and the study of crop or animal management practices is of great archaeological interest. Pickard et al. investigated animal foraging through carbon and stable isotope analysis of domesticates from the Late Chalcolithic site of Çamlıbel Tarlası (Turkey). Differences in isotopic values between cattle and other domesticates (pigs and caprines) were interpreted as a difference in feed or in feeding areas. Marciniak et al. compared carbon and nitrogen isotope ratios measured in bone collagen and oxygen and strontium isotope ratios measured in tooth enamel on LBK and TRB domesticates from Kopydłowo (Poland). Higher isotopic diversity observed for TRB cattle was interpreted as a greater variety of herding practices and landscape exploitation.

The overview given above of the contents of this special issue illustrates the importance and dynamics of isotopic studies in archaeological research. It is expected that this will continue to grow under novel methodological developments and the widening of research questions. 